\title{
Mammalian Spindle Orientation and Position Respond to Changes in Cell Shape in a Dynein- dependent Fashion $\mathbf{v}$
}

\author{
Christopher B. O'Connell and Yu-li Wang*
} Department of Physiology, University of Massachusetts Medical School, Worcester,
Massachusetts 01655

Submitted September 9, 1999; Revised March 2, 2000; Accepted March 3, 2000

Monitoring Editor: J. Richard McIntosh

\begin{abstract}
In animal cells, positioning of the mitotic spindle is crucial for defining the plane of cytokinesis and the size ratio of daughter cells. We have characterized this phenomenon in a rat epithelial cell line using microscopy, micromanipulation, and microinjection. Unmanipulated cells position the mitotic spindle near their geometric center, with the spindle axis lying roughly parallel to the long axis of the cell. Spindles that were initially misoriented underwent directed rotation and caused a delay in anaphase onset. To gain further insight into this process, we gently deformed cells with a blunted glass needle to change the spatial relationship between the cortex and spindle. This manipulation induced spindle movement or rotation in metaphase and/or anaphase, until the spindle reached a proper position relative to the deformed shape. Spindle positioning was inhibited by either treatment with low doses of nocodazole or microinjection of antibodies against dynein, apparently due to the disruption of the organization of dynein and/or astral microtubules. Our results suggest that mitotic cells continuously monitor and maintain the position of the spindle relative to the cortex. This process is likely driven by interactions among astral microtubules, the motor protein dynein, and the cell cortex and may constitute part of a mitotic checkpoint mechanism.
\end{abstract}

\section{INTRODUCTION}

Correct placement of the cleavage furrow is essential for the successful conclusion of mitosis and meiosis. During typical cell division, a centrally placed cleavage plane ensures that the two daughter cells receive a similar share of organelles and molecular components. During embryonic development, regulated asymmetric division coupled with localization of signaling molecules or organelles functions as an effective means for determining cell fate (Strome, 1993). Although it has been well established that the plane of cytoplasmic division is determined by the position of the mitotic spindle (reviewed by Fishkind and Wang, 1995; Glotzer, 1997; Field et al., 1999; Hales et al., 1999), little is known about how the location of the spindle itself is regulated.

$\nabla$ Online version of this article contains video material for Figures 1, 3, 4, 7, and 9. Online version available at www. molbiolcell.org.

*Corresponding author. E-mail address: yuli.wang@ umassmed.edu.

Abbreviations used: NA, numerical aperture; NRK, normal rat kidney.
What little evidence there is comes mostly from invertebrates and suggests that astral microtubules, which link the spindle with the cortex, are involved in bringing the spindle to defined sites within the cytoplasm (Shaw et al., 1997). In addition, studies with Saccharomyces cerivisae and embryos of Caenorhabditis elegans and Drosophila melanogaster indicate that dynein interacts with its accessory protein, dynactin, to generate forces for positioning the nucleus or mitotic spindle (Carminati and Stearns, 1997; McGrail and Hays, 1997; Skop and White, 1998). However, because little work has been done to identify an active spindle-positioning mechanism in other cell types, it was not clear whether these activities represent a general cellular function or specialized processes in yeast or large embryos to bring the spindle to specific destinations. Furthermore, although there is some evidence that molecular signals influence the position of the spindle (Strome, 1993), it is not known whether the location and orientation of the spindle are predetermined by a specific cortical region, as was suggested in yeast and C. elegans embryos (Snyder et al., 1991; Hyman and Stearns, 1992; Carminati and Stearns, 1997; Skop and White, 1998), or maintained by global interactions with the cortex. For cells without a predetermined polarity, the latter seems to be more advantageous for ensuring a proper spindle position and orientation. It is also unclear whether a preferred spin- 

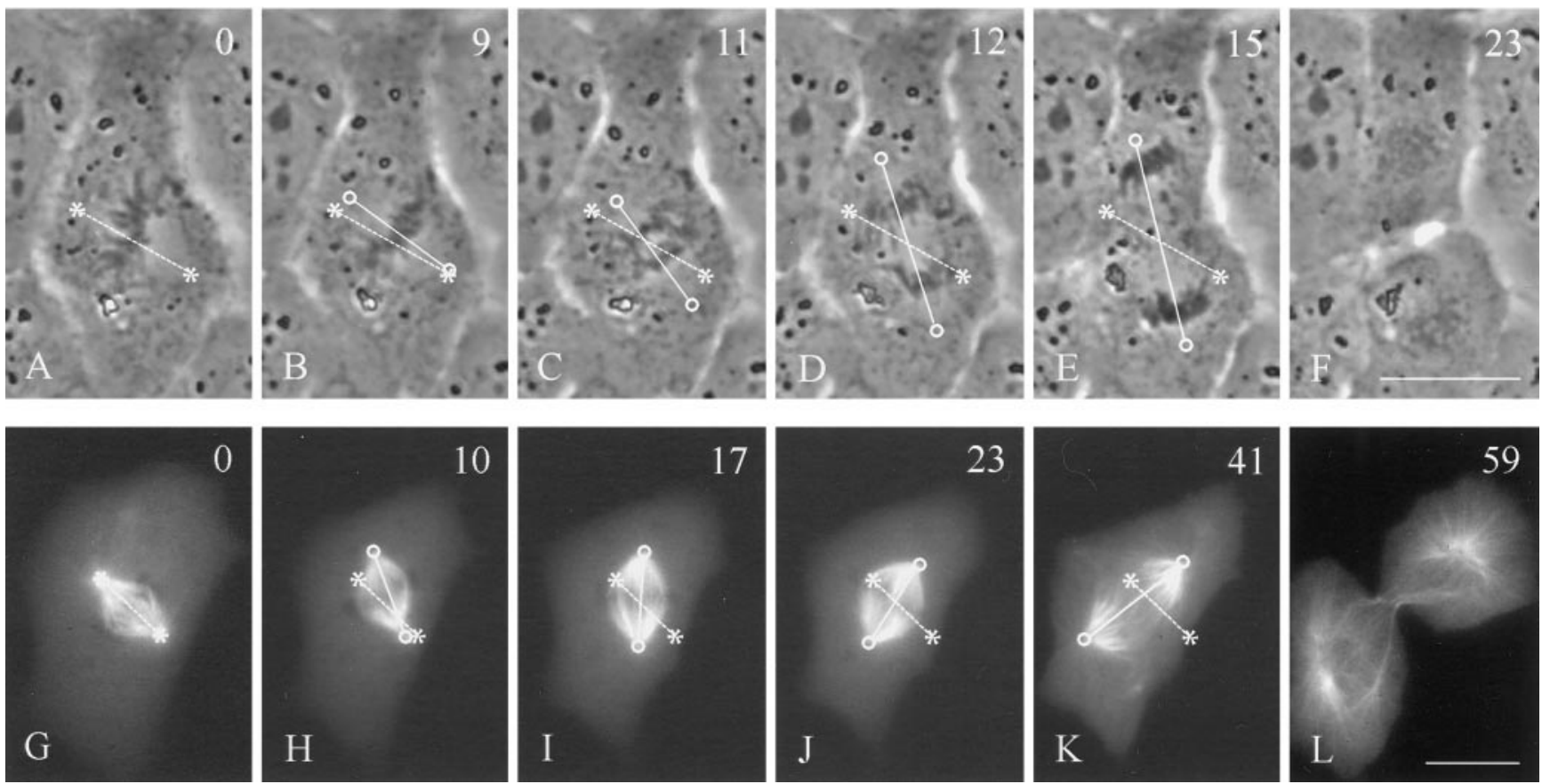

Figure 1. Rotation of mitotic spindles in unmanipulated NRK cells. Time-lapse phase-contrast images (A-F) or microinjected rhodaminetubulin images $(\mathrm{G}-\mathrm{L})$ were recorded in cells where the initial spindle orientation deviates from the long axis of the cell (A and $\mathrm{G})$. Rotation of the spindle in the upper cell took place mostly in anaphase (C-E). Spindle rotation in the lower cell took place during metaphase (H-J). Both reached an orientation roughly along the longest axis of the cell (E and $\mathrm{K}$ ). Time in minutes relative to metaphase is shown in the upper right corner. Asterisks and dotted lines indicate initial positions of the spindle poles and axis, respectively. Circles and solid lines indicate current positions of the spindle poles and axis, respectively. Bars, $20 \mu \mathrm{m}$.

dle position persists throughout mitosis or whether the cell continuously monitors and adjusts the position of the spindle relative to the cortex.

In this study, we started with observations of the spindle in unperturbed cultured normal rat kidney (NRK) cells to determine whether there is a preferred spindle location and orientation and whether cells are capable of correcting spontaneous mispositioning that may occur early during mitosis. We then developed a novel micromanipulation approach to alter the geometric relationship between the mitotic spindle and the cell cortex to determine whether cells are capable of responding to such challenges. This manipulation, along with microinjection and drug treatments, allowed us to probe into the mechanism of spindle relocation while optical sectioning and image reconstruction provided detailed structural data. Our results indicate that cultured mammalian cells do possess an active surveying mechanism for positioning the spindle. The spindle appears to be in constant communication with the cortex throughout much of mitosis, maintaining a central location in the cell and an orientation along the cellular long axis despite drastic changes in cell shape. Furthermore, the mechanism is dependent on dynein localized along astral microtubules.

\section{MATERIALS AND METHODS}

\section{Cell Culture, Microscopy, and Data Collection}

NRK cells (NRK-52E; American Type Culture Collection, Manassas, VA) were cultured on glass coverslips as previously described
(McKenna and Wang, 1989). This subclone maintains a particularly well-spread morphology throughout mitosis, making it ideal for micromanipulation and for visualization of the chromosomes and spindle. Cells were maintained in Kaighn's modified F-12 medium (Sigma, St. Louis, MO) supplemented with $10 \%$ fetal calf serum (JRH Biosciences, Lenexa, KS), $50 \mu \mathrm{g} / \mathrm{ml}$ streptomycin, $50 \mathrm{U} / \mathrm{ml}$ penicillin, and $1 \mathrm{mM}$ L-glutamine.

Imaging was performed on an Axiovert S100 TV inverted microscope (Carl Zeiss, Thornwood, NY) with a $40 \times$, numerical aperture (NA) $0.65 \mathrm{CP}$-achromat phase contrast lens, a $40 \times$, NA 0.75 planachroplan lens, or a $100 \times$, NA 1.3 Fluar lens. All images were acquired with a cooled charge-coupled device camera (CH250; Photometrics, Tucson, AZ; or NTE/CCD-512 EBFT; Roper Scientific, Trenton, NJ) and processed with custom software. Optical sectioning, deconvolution, and image reconstruction for the $90^{\circ}$ view were performed as described elsewhere (Fishkind and Wang, 1993; Wang, 1998).

\section{Micromanipulation and Microinjection}

The shape of mitotic cells was deformed using the tip of a blunted microneedle. Glass capillary tubing with an outer diameter of 1.2 $\mathrm{mm}$ and inner diameter of $0.9 \mathrm{~mm}$ was pulled into needles with a vertical micropipette puller (David Kopf Instruments, Tujunga, CA). The tips were then melted and shaped using a microforge (Narishige, East Meadow, NY). With a micromanipulater (Leitz, Wetzlar, Germany) the blunted needle tip was placed against the membrane and gently maneuvered to deform the shape of the cell.

Monoclonal antibody to dynein intermediate chain (clone 70.1; Sigma) was received as ascites fluid and prepared for microinjection by dialyzing overnight into a buffer containing $50 \mathrm{mM}$ potassium glutamate and $0.5 \mathrm{mM} \mathrm{MgCl} 2, \mathrm{pH}$ 6.7. The antibody was concen- 



Figure 2. Time-lapse images of control manipulation performed on metaphase cells. The membrane was pushed perpendicular to the cell axis near the metaphase plate (B), causing no change in the orientation of the long axis of the cell. No change in the position or orientation of the spindle took place, and both anaphase and cytokinesis progressed normally (B-E). Time in minutes relative to metaphase is shown in the upper right corner. Asterisks and dotted lines indicate initial positions of the spindle poles and axis, respectively. Circles and solid lines indicate current positions of the spindle poles and axis, respectively. Bar, $20 \mu \mathrm{m}$.

trated with a Microcon-30 centrifugal microconcentrator (Amicon, Beverly, MA) to a total protein concentration of $20-50 \mathrm{mg} / \mathrm{ml}$. As a control experiment mouse immunoglobulin $\mathrm{M}$ ascites fluid (Sigma) was prepared identically to a concentration of $21 \mathrm{mg} / \mathrm{ml}$. Rhoda- mine tubulin was prepared as described previously (Wheatley and Wang, 1996), and microinjection was performed as described by Wang (1992).

\section{Drug Treatment and Immunofluorescence}

Nocodazole (Sigma) was kept as a frozen $200 \mu \mathrm{M}$ stock solution in DMSO and diluted into culture media to a final concentration of 100 $\mathrm{nM}$. Cells were treated with the drug for $>24 \mathrm{~h}$ as described previously by Jordan et al. (1992).

Dynein staining was performed with a method modified from those of Busson et al. (1998) and Keith (1991). Cells were washed twice with warm PBS and then extracted for 1 min in PHEM buffer (100 mM 1,4-piperazinediethanesulfonic acid, $25 \mathrm{mM}$ HEPES, $1 \mathrm{mM}$ EGTA, and $2 \mathrm{mM} \mathrm{MgCl}_{2}, \mathrm{pH} 7.0$ ) containing $0.5 \%$ Triton X-100 and $5 \mu \mathrm{M}$ taxol (Paclitaxel; Sigma) to preserve microtubules while removing tubulin dimers. This was followed by fixation for $5 \mathrm{~min}$ in methanol chilled to $-20^{\circ} \mathrm{C}$. The intermediate chain of dynein was stained with L5 polyclonal antiserum (a gift from R. Vallee, University of Massachusetts Medical School; and K. Vaughn, University of Notre Dame, South Bend, IN) at a dilution of 1:750, followed by Alexa 488 conjugated goat anti-rabbit secondary antibody (Molecular Probes, Eugene, OR) diluted 1:100.

Fixation of NRK cells for microtubule immunofluorescence was performed as described previously (O'Connell et al., 1999). Tubulin was stained with anti- $\alpha$-tubulin mAb (clone DM 1A; Sigma) at a dilution of 1:25 and Alexa 546 conjugated goat anti-mouse antibody (Molecular Probes) at a dilution of 1:100.

To visualize chromosomes cells were incubated for $30 \mathrm{~min}$ with Hoechst 33258 (Sigma) diluted 1:1000 in PBS from a $10 \mathrm{mg} / \mathrm{ml}$ stock solution in DMSO

\section{RESULTS}

\section{Mitotic Spindles Orient along the Long Axis of Cultured Cells}

We started by observing the positioning of the mitotic spindle in a population of unperturbed NRK cells. Although the spindle was always located near the geometric center of the cell as early as prometaphase, in many cases the spindle axis was misoriented with respect to the long axis of the cell during prometaphase and/or metaphase. From time-lapse recording of phase images or microinjected rhodamine-tubulin, it became clear that misorientation was corrected by directed rotation of the spindle (Figure 1). Rotation took place in metaphase and/or anaphase and stopped once the spindle became roughly parallel to the cellular long axis. Only $35 \%$ of dividing cells initially formed a spindle orientated along the long axis $(n=26$; Figure 1 , A and G; a spindle axis within $20^{\circ}$ of the long axis was considered correct). However, by the end of metaphase, $62 \%$ of the spindles became parallel to the long axis, and by late anaphase, $81 \%$ of the spindles were aligned.

Although anaphase could start without the spindle oriented along the long axis, we found that there was a statistically significant $(\mathrm{p}<0.05)$ delay of anaphase onset in cells with misaligned spindles. Under our observation conditions, the length of metaphase (defined as the period between chromosome congregation and anaphase onset) was $4.8 \pm 1.2 \mathrm{~min}$ for cells that formed spindles with a correct orientation during prometaphase versus $8.5 \pm 2.3 \mathrm{~min}$ for those in which orientation was corrected at some point. These results suggest that there is an active mechanism for orienting the spindle along a preferred direction, and that a 

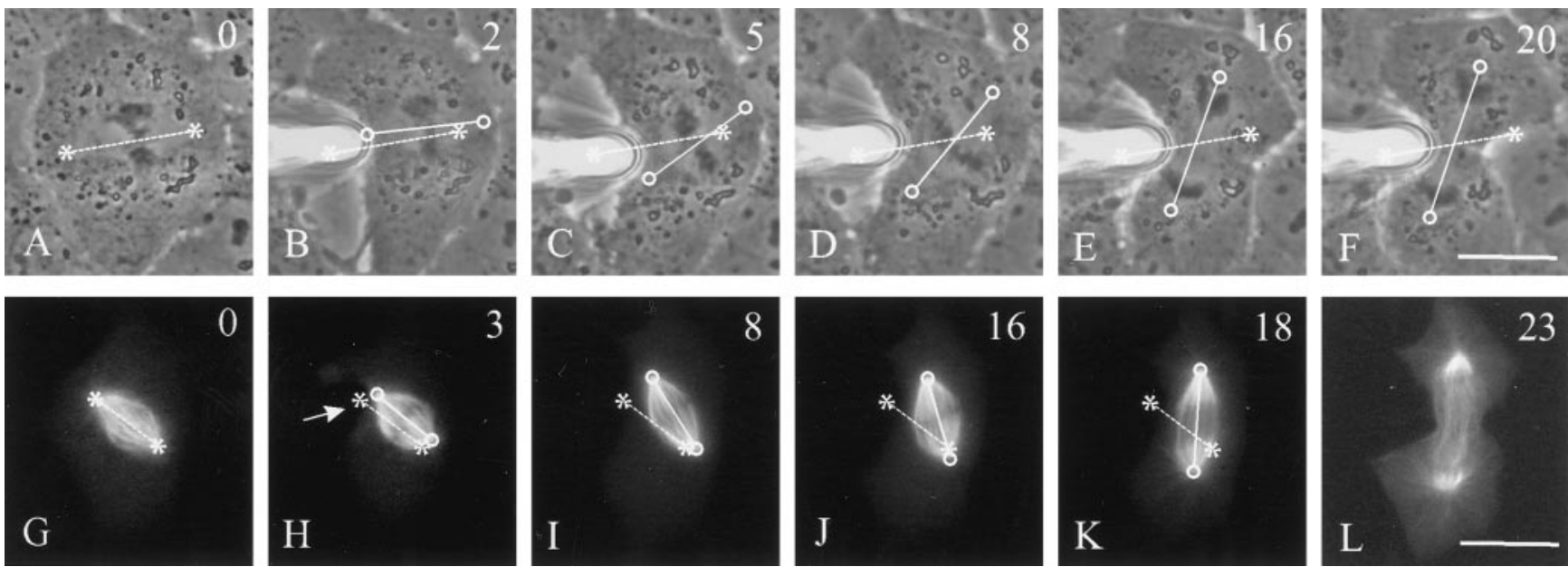

Figure 3. Rotation of mitotic spindle in a cell manipulated to alter the orientation of the long axis. A blunted microneedle was gently placed against the membrane and moved forward to push the cortex (B). Soon after, deformation spindles began to rotate (C-D). By mid-anaphase, the spindle was aligned along the longest axis of the cell (E). The same approach was also used to deform the cortex of a cell microinjected with rhodamine-labeled tubulin $(\mathrm{G}-\mathrm{L})$, which had a shape similar to that for the cell in A-F. The position of the needle is shown with an arrow $(\mathrm{H})$. The spindle rotated and the cell proceeded through anaphase and telophase, with no apparent damage to the spindle apparatus. Time relative to metaphase is shown in the upper right corner. Asterisks and dotted lines indicate initial positions of the spindle poles and axis, respectively. Circles and solid lines indicate current positions of the spindle poles and axis, respectively. Bars, $20 \mu \mathrm{m}$.

weak checkpoint regulates anaphase onset based on the orientation of the spindle.

\section{Mitotic Spindles Reposition in Response to Changes in Cell Shape}

The above observations imply that the spindle can actively detect the overall shape of the cell. To address this possibility, we used a blunted microneedle to deform the cell, by gently pushing the cortex in selected regions for a distance of 7-16 $\mu \mathrm{m}$. When cells with a properly positioned metaphase spindle were deformed without inducing spindle misalignment or mispositioning, no spindle movement was observed, and both mitosis and cytokinesis appeared indistinguishable from those in unperturbed cells (Figure 2). The deformation was then performed on cells in such a way as to create, or exaggerate, misorientation between the spindle axis and the long axis of the deformed cell (Figure 3). In most cases this was achieved by pushing the membrane near one of the spindle poles. Rotation of the spindle began as soon as 1 min after cortical deformation in some cells, reaching a correct orientation in $100 \%$ of the manipulated cells by late anaphase ( $\mathrm{n}=14$; Figure 3, E and K). Rotation occurred most frequently at metaphase $(71 \%)$ but also took place during anaphase $(29 \%)$ as for unmanipulated cells. The manipulation caused no visible effect on the morphology of the mitotic spindle, as judged from spindles labeled with microinjected fluorescent tubulin (Figure 3, G-L).

The micromanipulation approach also allowed us to determine whether cells can maintain the location of the mitotic spindle near its geometric center. This was not possible with unperturbed cells, because the spindle was always located near the center of the cell. We chose cells with an elongated morphology and deformed the membrane such that the spindle became much closer to one end of the cortex than the other end (Figure 4B, arrowhead). Within 1-2 min of deformation, the spindle began moving with an average rate of $0.5 \mu \mathrm{m} / \mathrm{min}$ toward the center of the main portion of the deformed cell (Figure 4, B-E). This behavior was seen in $71 \%(\mathrm{n}=21)$ of manipulated cells. Together, these results indicate that spindles can move in response to changes in cell shape, with the type and extent of movement determined by the geometric relationship between the spindle and the cortex.

\section{Dynein and Astral Microtubules Are Involved in Spindle Positioning}

We asked whether dynein, a motor molecule implicated in positioning the yeast and C. elegans mitotic spindle, is required for spindle positioning. The distribution of dynein in dividing NRK cells was first examined in detail by immunofluorescence combined with optical sectioning, image deconvolution, and image reconstruction (Figure 5). During early prometaphase, dynein was localized at kinetochores of the condensed chromosmomes (Figure 5A). By late prometaphase, the distribution shifted to spindle poles and astral microtubules. Through metaphase and anaphase, dynein was localized predominantly along astral microtubules in a discontinuous manner (Figure 5, C and D), with some staining also appearing along interzonal microtubules during anaphase. The general pattern of dynein distribution was similar to that reported for dynactin in Madin-Darby canine kidney cells (Busson et al., 1998). However, direct comparison of the distributions of dynein and injected fluorescent tubulin indicated that dynein was not evenly distributed among all microtubules but was heavily enriched along astral microtubules (Figure 6, A-C).

As reported previously, nanomolar concentrations of nocodazole can induce a range of disrupted spindle morphol- 

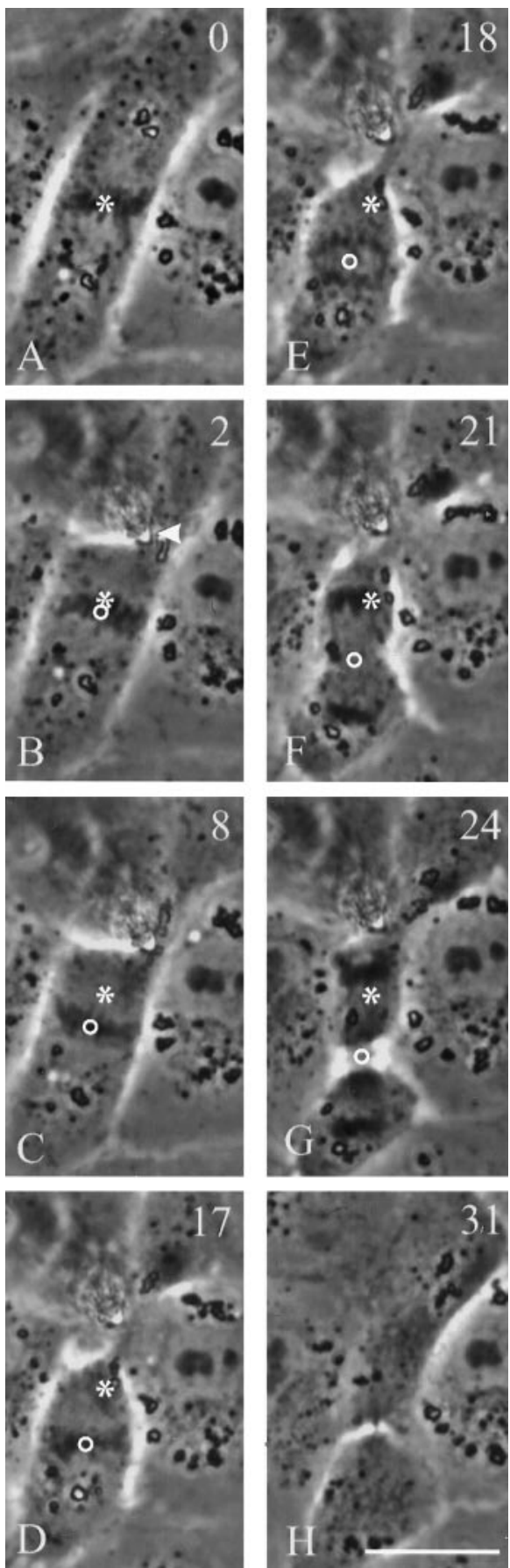

Figure 4. Spindle translocation in a cell manipulated to alter the position of the cell center. The cortex of this elongated cell was constricted near one end, such that the cortex became much closer to the upper spindle pole than to the lower spindle pole (B, arrowhead). The spindle gradually moved through the cytoplasm to a central position within the main cell mass (B-E). A cleavage furrow formed between the chromosomes at the center of the cell. The constriction disappeared upon removal of the needle, generating two daughter cells of unequal sizes $(\mathrm{H})$. Time relative to metaphase is shown in the upper right corner. Asterisks indicate the center of the metaphase plate before manipulation. Circles denote the current central position of the spindle. Bar, $20 \mu \mathrm{m}$. ogies (Jordan et al., 1992). We found that treatment of cells with $100 \mathrm{nM}$ nocodazole induced frequent misalignment and/or mispositioning of the mitotic spindle (Figure 7), although nocodazole concentrations at $\leq 50 \mathrm{nM}$ were not effective. Spindles in many treated cells maintained a relatively normal phase appearance and proceeded through metaphase and anaphase. The only clear differences of these spindles from control spindles were a shorter length (Figure $6 \mathrm{D})$, a slower pace of mitosis, and a reduced distance of chromosomal separation (Figure 7D). However, dynein in these cells showed a dramatically reduced localization along astral microtubules (Figure 6, E and F). In addition, careful examination of enhanced images suggested that the number and length of astral microtubules were greatly reduced in nocodazole-treated cells (Figure 6, compare A and D, insets; because of the much larger size of kinetochore bundles than single microtubules, astral microtubules in metaphase NRK cells were difficult to image).

To further test the functional role of dynein in spindle positioning, we microinjected the 70.1 monoclonal antibody against its intermediate chain, an approach used effectively in several studies (Vaisberg et al., 1993; Burkhardt et al., 1997). Although earlier studies indicated that dynein antibodies can inhibit spindle formation when injected during prophase, we found that injection of the 70.1 antibody during late prometaphase or early metaphase caused no apparent effect on the integrity of the spindle or anaphase chromosomal movement (Figure 8B'). Staining of 70.1-injected cells with polyclonal antibodies against the dynein intermediate chain revealed that dynein was removed from the astral microtubules and was visible only at the spindle poles (Figure 8A', arrows).

When injected cells were manipulated to induce and exaggerate misalignment (Figure 9, A-E), the spindles maintained their original orientation throughout metaphase and anaphase, causing separated chromosomes to become abnormally close to the cortex. In the cell shown in Figure 9, the plane of cytokinesis remained aligned with the spindle midplane, such that the cleavage furrow formed along the long axis of the cell. When antibody-injected cells were manipulated to cause incorrect spindle positioning as in Figure 4, the mitotic spindle remained mispositioned relative to the cell center, such that the cell divided into two unequal daughter cells (Figure 9, F-I). Inhibition of spindle movement (rotation or translation) was observed in $64 \%$ of cells injected with the 70.1 antibody $(n=11)$ compared with $25 \%$ of cells injected with control ascites fluid $(n=8)$ and $17 \%$ of uninjected, manipulated cells.

\section{DISCUSSION}

Although spindle positioning has been studied in yeast, $C$. elegans embryos, and D. melanogaster embryos (White and Strome, 1996; Carminati and Stearns, 1997; McGrail and Hays, 1997; Skop and White, 1998), it remains unclear whether this represents a process of general significance in mammalian cells. In this study, we used a novel micromanipulation approach to examine spindle positioning in cultured epithelial cells. It is clear from our results that NRK cells constantly monitor the spatial relationship between the mitotic spindle and the cell cortex, repositioning or reorienting the spindle upon any cell shape change that alters the 

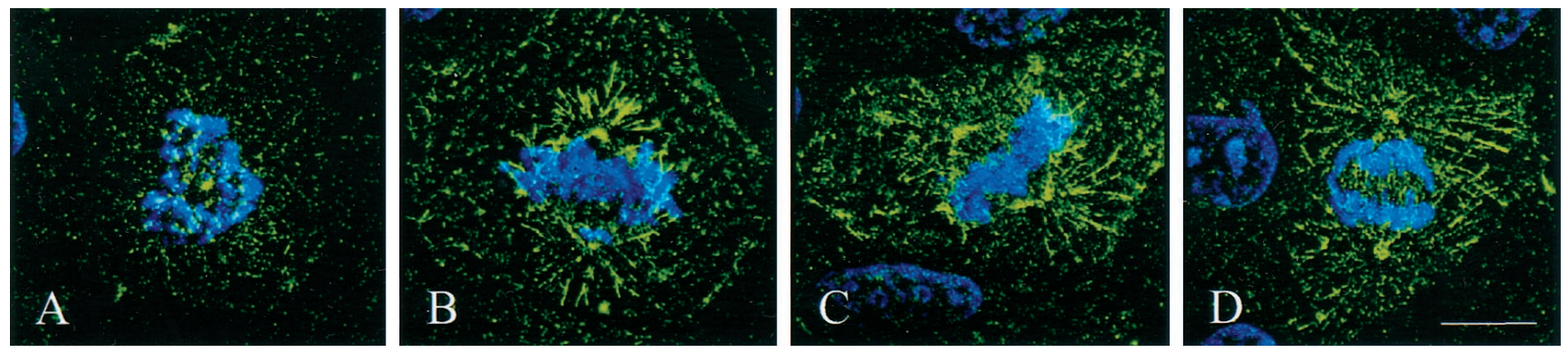

Figure 5. Pattern of dynein localization during mitosis. NRK cells were fixed and stained for the intermediate chain of dynein (green) and chromosomes (blue). The cells were optically sectioned, and images were deconvolved and reconstructed to show the $90^{\circ}$ view of structures on all focal planes. During early prometaphase (A), dynein is localized primarily at the kinetochores of the chromosomes. By late prometaphase (B), the distribution has changed dramatically. Dynein is now visible primarily on spindle poles and astral microtubules. Throughout metaphase and early anaphase (C and D), dynein is predominantly localized in a discontinuous pattern along astral microtubules. Some localization was also observed along interzonal microtubules. Bar, $10 \mu \mathrm{m}$.

geometric relationship between the spindle and the cell cortex. Because repositioning occurs in both unmanipulated and manipulated cells, but only when the geometric relationship between the spindle and the cortex deviates from the norm, this process most likely reflects a physiological event rather than a manipulation-induced response.

Spindle positioning in NRK cells follows a simple set of rules. The axis of the spindle is maintained along an axis where rotation does not cause a significant gain in the distance between the spindle and the cortex. In addition, the center of the spindle is always located near the geometric center of the cell.
Although anaphase can take place before the spindle reaches its final position, the onset of anaphase is significantly delayed in cells containing an incorrectly aligned spindle, suggesting that spindle positioning functions as a weak mitotic checkpoint. A spindle-positioning checkpoint has also been described recently in the yeast $S$. cerevisiae (Muhua et al., 1998). Although the mechanism is unclear, it is possible that the position of the spindle affects mechanical signals on the kinetochores, which control the chromosomal congregation checkpoint through a force-sensitive phosphorylation-dephosphorylation mechanism (Nicklas et al., 1995).

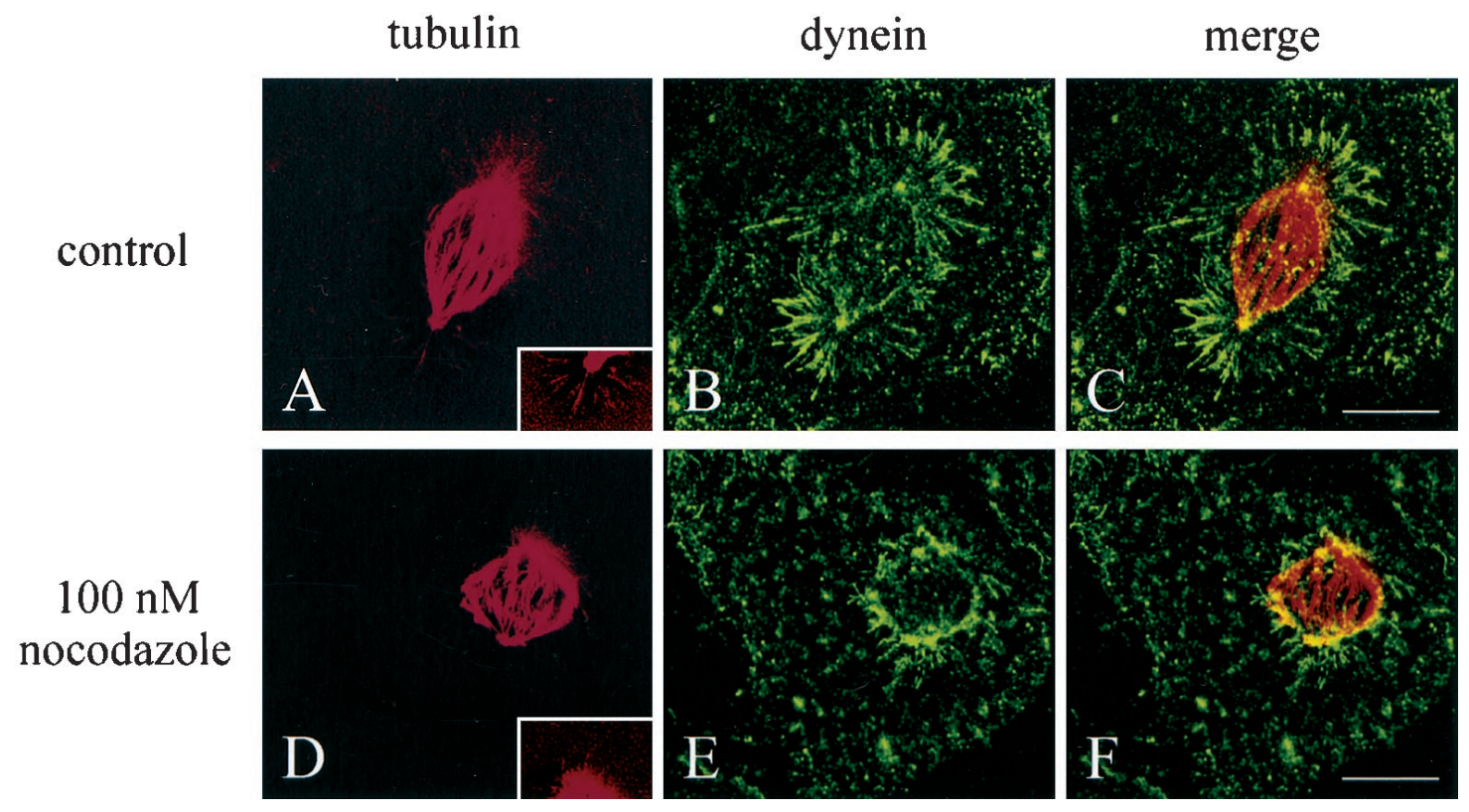

Figure 6. Organization of dynein and microtubules in a control cell (A-C) and a cell treated overnight with $100 \mathrm{nM}$ nocodazole (D-F). The cells were microinjected with fluorescent tubulin at prometaphase and fixed and stained at metaphase. Stacks of optical sections were deconvolved and reconstructed into the $90^{\circ}$ view. Microtubules in the mitotic spindle of the treated cell (D) had a typical bipolar arrangement, similar to those in control cells (A). However, both the spindle length and the number and length of astral microtubules are reduced. Enhanced views of astral microtubules are shown in the insets of A and D. The distribution of dynein also appeared disorganized after nocodazole treatment, with no accumulation where the astral microtubules would be (E and F), as seen in control cells (B and $\mathrm{C})$. Bars, $10 \mu \mathrm{m}$. 

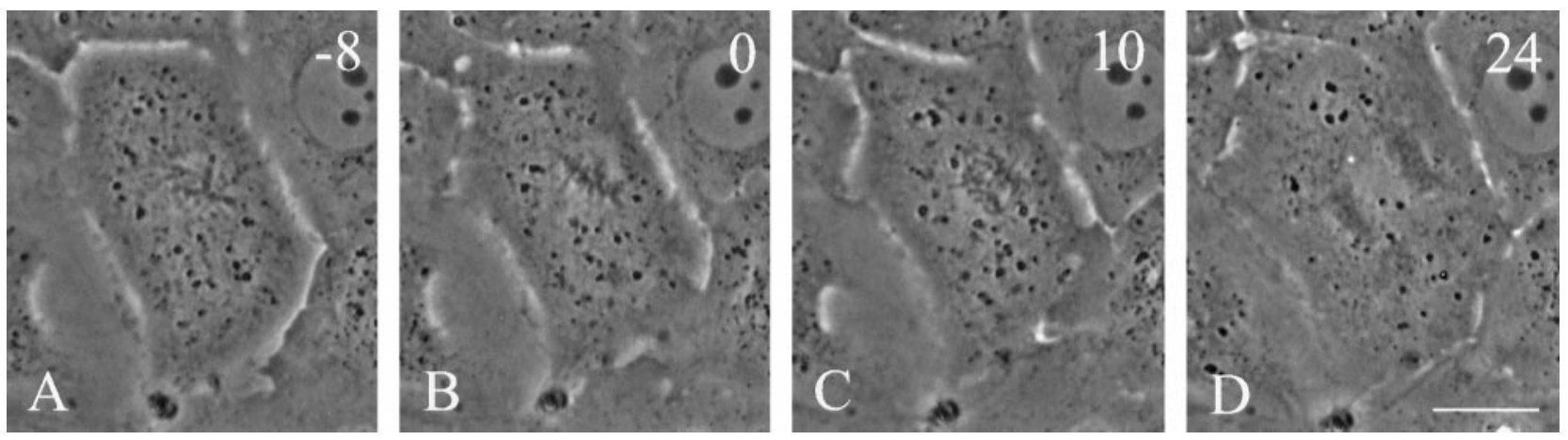

Figure 7. Time-lapse images of a dividing NRK cell treated with $100 \mathrm{nM}$ nocodazole for $24 \mathrm{~h}$. The metaphase spindle appeared normal under phase optics but was located away from the geometric center of the cell, with an orientation misaligned relative to the long axis of the cell (A and B). The cell proceeded into anaphase without correcting the position of the spindle (C). In this instance cytokinesis was inhibited, resulting in a binucleated cell (D). Time in minutes relative to metaphase is shown in the upper right corner. Bar, $20 \mu \mathrm{m}$.

Mechanistic insight into spindle positioning came first from immunofluorescence of dynein. We have obtained the most detailed images of dynein during mitosis to date by optical sectioning, image deconvolution, and image reconstruction, expanding on previous work with Madin-Darby canine kidney epithelial cells (Busson et al., 1998). Dynein moves from kinetochores to the spindle poles and astral microtubules during prometaphase. Given the minus end-
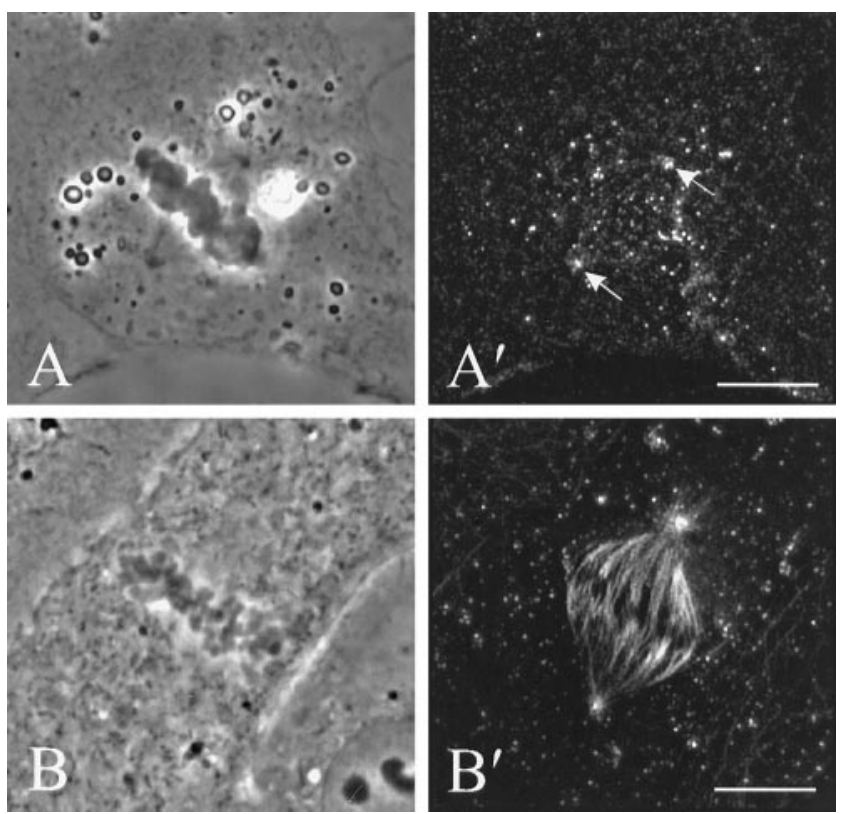

Figure 8. The organization of dynein $\left(\mathrm{A}^{\prime}\right)$ and microtubules $\left(\mathrm{B}^{\prime}\right)$ in NRK cells microinjected with the 70.1 monoclonal antibody against dynein intermediate chain. Stacks of optical sections were deconvolved and used for reconstruction of the $90^{\circ}$ view. Dynein staining revealed no localization along astral microtubules, although some staining is visible at the spindle poles (A and $\mathrm{A}^{\prime}$, arrows). The injection caused no apparent disruption of the spindle, as shown by immunofluorescence of tubulin in a separate cell (B and $\left.B^{\prime}\right)$. Bars, $10 \mu \mathrm{m}$. directed polarity of the dynein motor, this process must be achieved by either dissociation and reassociation of dynein or transport of dynein by a plus end-directed motor. One important aspect of this redistribution is that dynein is not evenly localized among all microtubules but is concentrated primarily along astral microtubules. This suggests that astral microtubules are involved in specific dynein-mediated mechanical events.

We found that spindle positioning can be inhibited by the injection of 70.1 anti-intermediate chain antibodies, an approach established previously for inhibiting the function of dynein (Vaisberg et al., 1993; Burkhardt et al., 1997), or by treatment with low doses of nocodazole. Furthermore, structural studies indicate that these treatments disrupt dynein localization along astral microtubules or the integrity of astral microtubules themselves. Together, these results strongly suggest that spindle positioning is an active process involving astral microtubules, dynein, and the cell cortex. The microtubule dependence of spindle positioning in culture mammalian cells is similar to that characterized in invertebrates and yeast. A "searching" mechanism has been proposed in yeast, where microtubule growth and shrinkage are required for successful migration of the nucleus to the mother-bud neck (Shaw et al., 1997). In addition, centrosomal rotation during early divisions in C. elegans is also dependent on microtubule dynamics (Hyman and White, 1987). However, unlike S. cerevisiae and C. elegans embryos, where there appears to be a defined target for spindle movement (reviewed by Strome, 1993), our results indicate that in NRK cells there is no fixed target position or orientation for the spindle. Instead, the spindle interacts continuously with the cortex searching for an optimal location and orientation throughout the period of mitosis.

Involvement of dynein in spindle positioning has also been implicated budding yeast and Drosophila embryos, where mutations of dynein cause defects in the localization of the spindle or partitioning of daughter nuclei (Eshel et al., 1993; Li et al., 1993; Yeh et al., 1995; Carminati and Stearns, 1997; McGrail and Hays, 1997). Moreover, a discrete cortical site on C. elegans embryos contains a concentration of dynactin and appears to direct spindle orientation (Skop and White, 1998). However, data from mutant strains of the 

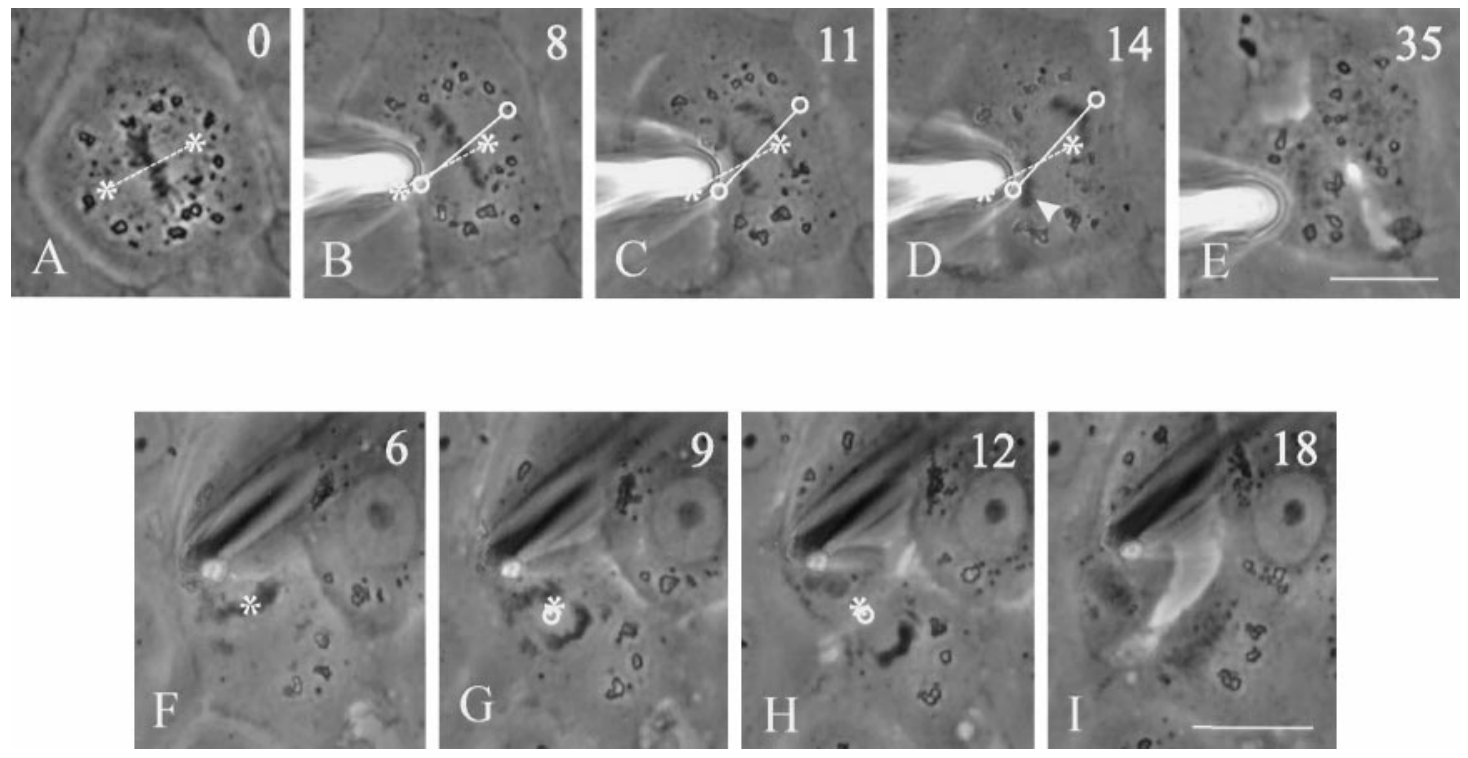

Figure 9. Inhibition of spindle rotation and migration by anti-dynein antibodies. Two NRK cells were injected with 70.1 antibodies against the dynein intermediate chain and deformed with a microneedle as in Figures 3 and 4 . In the first cell (A-E), the needle created a highly asymmetric cell shape. Throughout metaphase and anaphase the spindle remained close to its original orientation (B-D), such that one set of chromosomes bumped into the needle ( $\mathrm{D}$, arrowhead). A cleavage furrow developed along the longest axis of the cell between separating chromosomes. Asterisks and dotted lines indicate the initial positions of the spindle poles and axis, respectively. Circles and solid lines indicate current positions of the spindle poles and axis, respectively. In the second cell (F-I), the needle was used to create a constriction near one end of the cell, such that the cortex became much closer to one of the spindle poles. No repositioning of the spindle was detected. A cleavage furrow developed between separating chromosomes, creating two daughter cells of unequal sizes. Time relative to metaphase is shown in the upper right corner. Bars, $20 \mu \mathrm{m}$.

fission yeast Schizosaccharomyces pombe do not support this role for dynein in spindle positioning and orientation (Yamamoto et al., 1999). Therefore, the mechanism may not be a universal one. We also cannot rule out the possibility that other motor proteins may play a role in mammalian spindle dynamics, as has been shown to be the case in yeast (DeZwaan et al., 1997).

Given the strong evidence for the interaction between microtubules and dynein and the cortex, important questions remain as to how such interaction leads to the positioning of the spindle at the cell center and along the long axis. There are indications that the dynein-dynactin complex may be anchored to the cortex and capture microtubules, generating pulling force by moving toward the spindle pole (Carminati and Stearns, 1997; Busson et al., 1998). In addition, the magnitude of forces may be proportional to the length of the microtubules along the cortex, because longer microtubules may interact with more cortical dynein molecules. These forces provide an effective means for positioning the spindle, if they are exerted on microtubules that connect the spindle poles to the cortex within a finite angular span (Figure 10). For example, when the location of the spindle deviates from the cell center, pulling forces toward the proximal side of the cortex become weaker than those toward the opposite side, causing the spindle to migrate toward a central location. Similarly, a net torque would develop if microtubules are of unequal lengths on the two sides of the spindle axis. The torque should rotate the spindle toward an orientation that is parallel with the long axis (Figure 10A). Implied in this model is that at least a portion of astral microtubules interacts directly with the cortex. In addition, it suggests that dynein is active throughout the cortex of NRK cells but is localized at a defined cortical site in yeast and C. elegans embryos to define a specific spindle orientation or position. A similar mechanism involving microtubules and dynein may also function in the positioning of centrosomes and nuclei during interphase (reviewed by Reinsch and Gönczy, 1998; Schliwa et al., 1999).

Spindle positioning likely plays an important role in the division of all animal cells. A centrally located spindle would ensure that cells divide into daughter cells of similar sizes. In addition, an orientation along the long axis of the cell would ensure that cleavage occurs along the shortest dimension of the cell, which should be more efficient and less prone to disruptions. In polarized epithelial cells, the orientation of the spindle ensures cleavage parallel to the monolayer and prevents disruptions to the integrity of the membrane barrier (Reinsch and Karsenti, 1994). Finally, it is possible that dynein-membrane interactions for spindle positioning may also play a role in driving an equatorial bound cortical flow, which brings a number of membrane components toward the equator and may function in conjunction with signals from the central spindle to define the cleavage site.

\section{ACKNOWLEDGMENTS}

We thank Ruby Wang for performing the initial micromanipulation experiments and Drs. R. Vallee (University of Massachusetts Medical School) and K. Vaughn (University of Notre Dame) for L5 

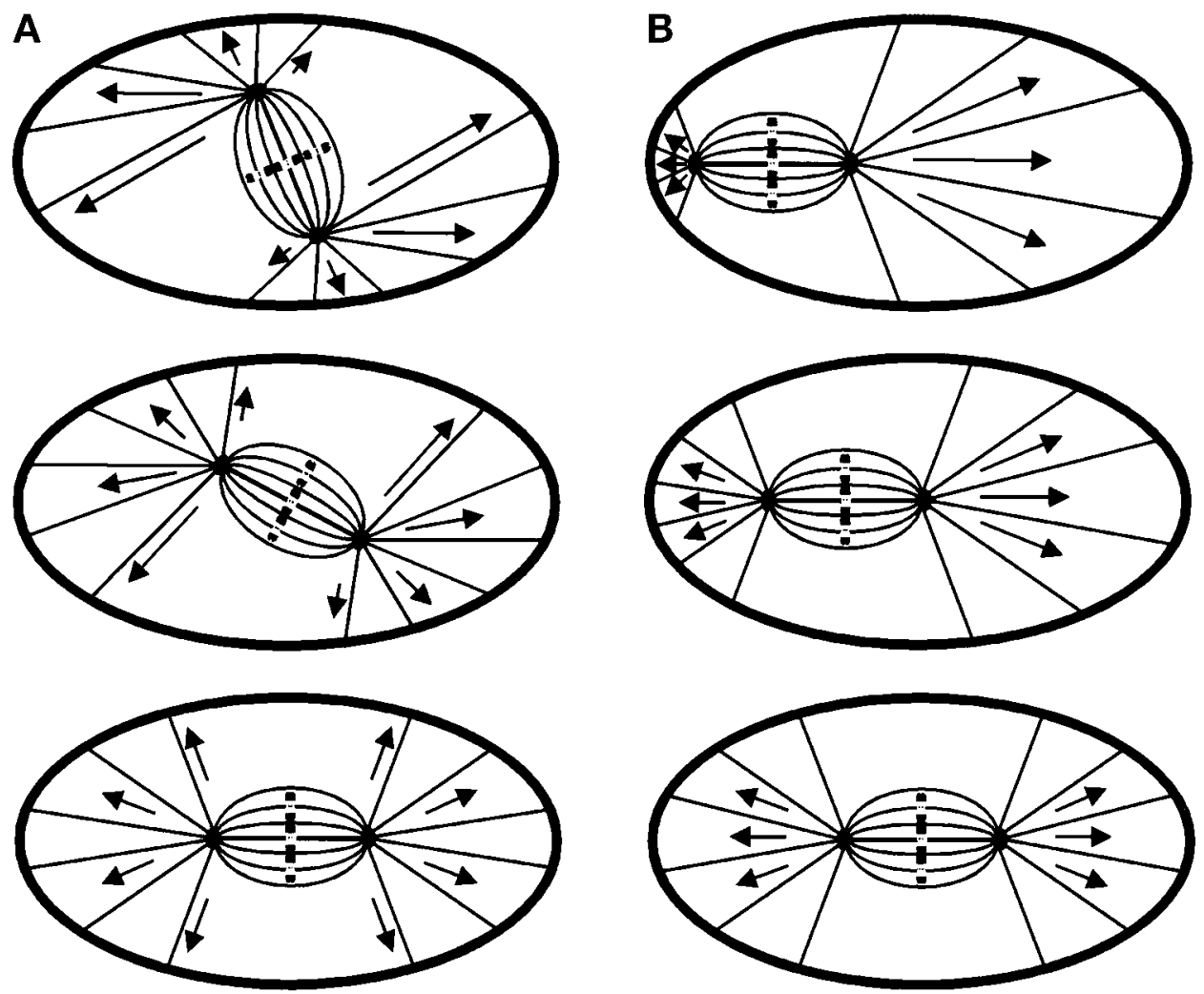

Figure 10. Models for spindle positioning in mitotic mammalian cells based on pulling forces exerted on astral microtubules. (A) When the spindle is misaligned relative to the long axis of the cell, the lengths of astral microtubules become asymmetric with respect to the spindle axis. Longer microtubules, which interact with more cortically associated dynein, exert stronger forces on the centrosomes and generate a torque toward the long axis of the cell. The forces become symmetric once the spindle is aligned with the long cellular axis, eliminating the torque. (B) A similar mechanism may also center the spindle at the geometric center of the cell. Shorter astral microtubules on the side of the spindle proximal to the cortex exert weaker forces than do longer ones on the opposite side. This produces a net force toward the distal cortex until the spindle reaches a central location.

polyclonal antiserum to the intermediate chain of dynein. We are also grateful to the Vallee group (University of Massachusetts Medical School) for helpful discussions. This research was supported by National Institutes of Health grant GM-32476.

\section{REFERENCES}

Burkhardt, J.K., Echeverri, C.J., Nilsson, T., and Vallee, R.B. (1997). Overexpression of the dynamitin (p50) subunit of the dynactin complex disrupts dynein-dependent maintenance of membrane organelle distribution. J. Cell Biol. 139, 469-484.

Busson, S., Dujardin, D., Moreau, A., Dompierre, J., and DeMey, J.R. (1998). Dynein and dynactin are localized to astral microtubules and at cortical sites in mitotic epithelial cells. Curr. Biol. 8, 541-544.

Carminati, J.L., and Stearns, T. (1997). Microtubules orient the mitotic spindle in yeast through dynein-dependent interactions with the cell cortex. J. Cell Biol. 138, 629-641.

DeZwaan, T.D., Ellingson, E., Pellman, D., and Roof, D.M. (1997). Kinesin-related KIP3P of Saccharomyces cerevisiae is required for a distinct step in nuclear migration. J. Cell Biol. 138, 1023-1040.

Eshel, D., Urrestarazu, L.A., Vissers, S., Jauniaux, J.C., Van-VlietReedijk, J.C., Planta, R.J., and Gibbons, I.R. (1993). Cytoplasmic dynein is required for normal nuclear segregation in yeast. Proc. Natl. Acad. Sci. USA 90, 11172-11176.

Field, C., Li, R., and Oegema, K. (1999). Cytokinesis in eukaryotes: a mechanistic comparison. Curr. Opin. Cell Biol. 11, 68-80.

Fishkind, D.J., and Wang, Y.-1. (1993). Orientation and three-dimensional organization of actin filaments in dividing cultured cells. J. Cell Biol. 123, 837-848.

Fishkind, D.J., and Wang, Y.-1. (1995). New horizons for cytokinesis. Curr. Opin. Cell Biol. 7, 23-31.

Glotzer, M. (1997). The mechanism and control of cytokinesis. Curr. Opin. Cell Biol. 9, 815-823.

Hales, K.G., Bi, E., Wu, J.-Q., Adam, J.C., Yu, I.-C., and Pringle, J.R. (1999). Cytokinesis: an emerging unified theory for eukaryotes? Curr. Opin. Cell Biol. 11, 717-725.

Hyman, A.A., and Stearns, T. (1992). Spindle positioning and cell polarity. Curr. Biol. 2, 469-471.

Hyman, A.A., and White, J.G. (1987). Determination of cell division axis in the early embryogenesis of Caenorhabditis elegans. J. Cell Biol. $105,2123-2135$.

Jordan, M.A., Thrower, D., and Wilson, L. (1992). Effects of vinblastine, podophyllotoxin and nocodazole on mitotic spindles: implica- 
tions for the role of microtubule dynamics in mitosis. J. Cell Sci. 102, 401-416.

Keith, C.H. (1991). Quantitative fluorescence techniques for the determination of local microtubule polymerization equilibria in cultured neurons. J. Neurosci. Methods 39, 141-152.

Li, Y.-Y., Yeh, E., Hays, T., and Bloom, K. (1993). Disruption of mitotic spindle orientation in a yeast dynein mutant. Proc. Natl. Acad. Sci. USA 90, 10096-10100.

McGrail, M., and Hays, T.S. (1997). The microtubule motor cytoplasmic dynein is required for spindle orientation during germline cell divisions and oocyte differentiation in Drosophila. Development 124, 2409-2419.

McKenna, N.M., and Wang, Y.-l. (1989). Culturing cells on the microscope stage. In: Methods in Cell Biology, vol. 29, ed. Y.-1. Wang and D.L. Taylor, New York: Academic Press, 195-205.

Muhua, L., Adames, N.R., Murphy, M.D., Shields, C.R., and Cooper, J.A. (1998). A cytokinesis checkpoint requiring the yeast homologue of an APC-binding protein. Nature 393, 487-491.

Nicklas, R.B., Ward, S.C., and Gorbsky, G.J. (1995). Kinetochore chemistry is sensitive to tension and may link mitotic forces to a cell cycle checkpoint. J. Cell Biol. 130, 929-939.

O'Connell, C.B., Wheatley, S.P., Ahmed, S., and Wang, Y.-1. (1999). The small GTP-binding protein Rho regulates cortical activities in cultured cells during division. J. Cell Biol. 144, 305-313.

Reinsch, S., and Gönczy, P. (1998). Mechanisms of nuclear positioning. J. Cell Sci. 111, 2283-2295.

Reinsch, S., and Karsenti, E. (1994). Orientation of spindle axis and distribution of plasma membrane proteins during cell division in polarized MDCKII cells. J. Cell Biol. 126, 1509-1526.

Schliwa, M., Euteneuer, U., Graf, R., and Ueda, M. (1999). Centrosomes, microtubules and cell migration. Biochem. Soc. Symp. 65, 223-231.
Shaw, S.L., Yeh, E., Maddox, P., Salmon, E.D., and Bloom, K. (1997). Astral microtubule dynamics in yeast: a microtubule-based searching mechanism for spindle orientation and nuclear migration into the bud. J. Cell Biol. 139, 985-994.

Skop, A.R., and White, J.G. (1998). The dynactin complex is required for cleavage plane specification in early Caenorhabditis elegans embryos. Curr. Biol. 8, 1110-1116.

Snyder, M.S., Gehrung, S., and Page, B.D. (1991). Studies concerning the temporal and genetic control of cell polarity in Saccharomyces cerevisiae. J. Cell Biol. 114, 515-532.

Strome, S. (1993). Determination of cleavage planes. Cell 72, 3-6.

Vaisberg, E.A., Koonce, M.P., and McIntosh, J.R. (1993). Cytoplasmic dynein plays a role in mammalian mitotic spindle formation. J. Cell Biol. 123, 849-858.

Wang, Y.-1. (1992). Fluorescence microscopic analysis of cytoskeletal organization and dynamics. In: The Cytoskeleton: A Practical Approach, ed. K.L. Carraway and C.A.C. Carraway, Oxford, United Kingdom: IRL Press, 1-22.

Wang, Y.-1. (1998). Digital deconvolution of fluorescence images for biologists. Methods Cell Biol. 56, 305-315.

Wheatley, S.P., and Wang, Y.-1. (1996). Midzone microtubule bundles are continuously required for cytokinesis in cultured epithelial cells. J. Cell Biol. 135, 981-989.

White, J., and Strome, S. (1996). Cleavage plane specification in C. elegans: how to divide the spoils. Cell 84, 195-198.

Yamamoto, A., West, R.R., McIntosh, J.R., and Hiraoka, Y. (1999). A cytoplasmic dynein heavy chain is required for oscillatory nuclear movement of meiotic prophase and efficient meiotic recombination in fission yeast. J. Cell Biol. 145, 1233-1250.

Yeh, E., Skibbens, R.V., Cheng, J.W., Salmon, E.D., and Bloom, K. (1995). Spindle dynamics and cell cycle regulation of dynein in the budding yeast Saccharomyces cerevisiae. J. Cell Biol. 130, 687-700. 